\title{
Determination of the bonding configuration of the metastable molecular oxygen adsorbed on a Si(111)-(7×7) surface
}

\author{
Kazuyuki Sakamoto, Fumihiko Matsui, Masumi Hirano, Han Woong Yeom, Hanmin Zhang \\ and Roger Uhrberg
}

\section{Linköping University Post Print}

\section{Tweet}

N.B.: When citing this work, cite the original article.

Original Publication:

Kazuyuki Sakamoto, Fumihiko Matsui, Masumi Hirano, Han Woong Yeom, Hanmin Zhang and Roger Uhrberg, Determination of the bonding configuration of the metastable molecular oxygen adsorbed on a Si(111)-(7×7) surface, 2002, Physical Review B. Condensed Matter and Materials Physics, (65), 20, .

http://dx.doi.org/10.1103/PhysRevB.65.201309

Copyright: American Physical Society

http://www.aps.org/

Postprint available at: Linköping University Electronic Press

http://urn.kb.se/resolve?urn=urn:nbn:se:liu:diva-47879 


\title{
Determination of the bonding configuration of the metastable molecular oxygen adsorbed on a $\mathrm{Si}(111)-(7 \times 7)$ surface
}

\author{
Kazuyuki Sakamoto, ${ }^{1,2, *}$ Fumihiko Matsui, ${ }^{3}$ Masumi Hirano, ${ }^{2}$ Han Woong Yeom, ${ }^{4}$ H. M. Zhang, ${ }^{1}$ and R. I. G. Uhrberg ${ }^{1}$ \\ ${ }^{1}$ Department of Physics and Measurement Technology, Linköping University, S-581 83 Linköping, Sweden \\ ${ }^{2}$ Department of Physics, Graduate School of Science, Tohoku University, Sendai 980-8578, Japan \\ ${ }^{3}$ Graduate School of Material Science, Nara Institute of Science and Technology, Ikoma, Nara 630-0101, Japan \\ ${ }^{4}$ Atomic-Scale Surface Science Research Center and Institute of Physics and Applied Physics, Yonsei University, Seoul 120-790, Korea
}

(Received 9 April 2002; published 14 May 2002)

\begin{abstract}
The initial adsorption stage of oxygen on a $\mathrm{Si}(111)-(7 \times 7)$ surface has been investigated using real time high-resolution core-level measurements at $100 \mathrm{~K}$. Among the six $\mathrm{O} 1 \mathrm{~s}$ components observed on an $\mathrm{O}_{2}$ exposed $\mathrm{Si}(111)$ surface, the three components, whose intensities saturate at the same dosage, have the same lifetime. These results lead to a conclusion that three oxygen atoms with different chemical environments compose the metastable species. Based on a comparison with our calculated $\mathrm{O} 1 \mathrm{~s}$ binding energies, we have determined the bonding configuration of the metastable oxygen.
\end{abstract}

DOI: 10.1103/PhysRevB.65.201309

PACS number(s): 68.43. - h, 71.15.Ap, 73.20.Hb, 79.60.-i

Adsorption and reaction of oxygen have been ongoing subjects of physical and chemical interest for the understanding of the oxidation processes of materials. Regarding silicon surfaces, a great deal of endeavor has been devoted to the atomic level understanding of their oxidation, due to the technological importance of nanoscale dielectric layers in semiconductor devices. Nevertheless, in spite of extensive research efforts, the initial oxidation stage on silicon surfaces is still controversial.

One of the most heated debates on the initial oxidation stage concerns the interpretation of the metastable oxygen adsorbed on a $\mathrm{Si}(111)-(7 \times 7)$ surface. In studies based on density-functional theory (DFT) calculations, ${ }^{1,2}$ it was stated that the metastable oxygen has an atomic oxygen configuration. These DFT calculations ${ }^{1,2}$ reinterpreted the earlier spectroscopic $^{3-10}$ and microscopic ${ }^{11-16}$ results in which the metastable oxygen was reported to be a molecular species, a so-called molecular precursor. The absence of the molecular precursor was supported by a Cs ion scattering experiment. ${ }^{17}$ However, quite recently, one theoretical ${ }^{18}$ and three spectroscopic ${ }^{19-21}$ studies have suggested the adsorption of metastable molecular oxygen on a $\mathrm{Si}(111)$ surface. These recent studies ${ }^{18-21}$ reported that the metastable molecular species adsorb on a $\mathrm{Si}(111)$ surface, on which oxygen atoms are already adsorbed, and they showed the necessity to consider at least three oxygen atoms with different chemical environments. This number is different from that used for the previous models, i.e., only two oxygen atoms with different chemical environments have been considered for the atomic configuration $^{1,2,17}$ and the molecular precursor. ${ }^{3-16}$ This obvious conflict calls for an urgent and careful reinvestigation of the structural model of the metastable species, which is essential for the understanding of the oxidation process of $\mathrm{Si}$ on an atomic level. Since the long debate principally originates from the uncertainty in the number of oxygen atoms composing the metastable species, a probe sensitive to the chemical environments of the oxygen atoms is required.

High-resolution core-level spectroscopy provides crucial information about the chemical environment of specific elements, and thus stringent spectroscopic criteria for a correct structural model. In this Rapid Communication, we present real time high-resolution $\mathrm{O} 1 \mathrm{~s}$ core-level measurements of oxygen adsorption and reaction on a $\mathrm{Si}(111)-(7 \times 7)$ surface at $100 \mathrm{~K}$. During oxygen exposure, we observed the development of six O $1 \mathrm{~s}$ components with binding energies of $527.3,530.4,531.7,532.3,533.2$, and $534.9 \mathrm{eV}$. The intensities of the 527.3-, 530.4-, and 534.9-eV components saturate at the same dosage. After stopping the exposure, these three components show the same lifetime. These results suggest that three oxygen atoms with different chemical environments compose the metastable species. Taking our calculated $\mathrm{O} 1 s$ binding energies into account, the metastable oxygen is concluded to consist of a molecule adsorbed on top of a $\mathrm{Si}$ atom that has one back bond with an inserted oxygen atom. The present result provides a detailed atomic level understanding of the initial oxidation stage of a $\operatorname{Si}(111)-(7 \times 7)$ surface, and settles the debate on the bonding configuration of the metastable oxygen.

The high-resolution core-level photoemission measurements were performed at beamline I311 at the MAX-II synchrotron-radiation facility in Lund, Sweden. The O $1 \mathrm{~s}$ core-level spectra were obtained with an angle-integrated photoelectron spectrometer with a total energy resolution of $\sim 0.23 \mathrm{eV}$ at a photon energy of $665 \mathrm{eV}$, and an acceptance angle of $15^{\circ}$. The vacuum chamber was also equipped with a low-energy electron diffraction (LEED) system and a quadrupole mass spectrometer (QMS). The base pressure was below $4 \times 10^{-11}$ Torr. The Si(111) sample, cut from a Sb-doped (n-type) Si wafer, was annealed at $1520 \mathrm{~K}$ for $3 \mathrm{sec}$ in the vacuum chamber to obtain a clean surface. After the annealing, a sharp $7 \times 7$ LEED pattern was observed, and neither the $\mathrm{C} 1 s$ nor the $\mathrm{O} 1 s$ peak was detected in the core-level spectra. The $\mathrm{Si}(111)-(7 \times 7)$ sample was then cooled down to $100 \mathrm{~K}$ before the oxygen exposure for the following reasons. First, a short lifetime might cause a problem to obtain accurate information about the metastable oxygen due to the finite measuring time, and second, the lifetime is longer at a lower temperature. ${ }^{3}$ No additional $\mathrm{H}_{2} \mathrm{O}$ or $\mathrm{CO}$ was detected by QMS during the exposure to $\mathrm{O}_{2}$ gas. 


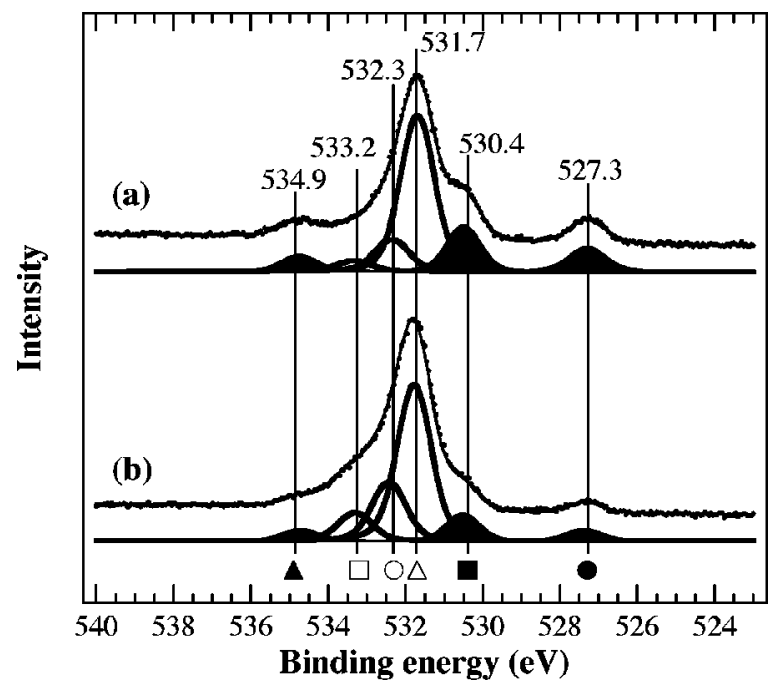

FIG. 1. O $1 s$ core-level spectra of the $20 \mathrm{~L} \mathrm{O}_{2}$ exposed $\mathrm{Si}(111)-(7 \times 7)$ surfaces, (a) is recorded $15 \mathrm{~min}$ after the $\mathrm{O}_{2}$ exposure, and (b) shows the spectrum recorded $205 \mathrm{~min}$ after the exposure. The dots are the experimental data, and the solid lines overlapped with the dots are the fitting results that are obtained using the components shown at the bottom of each spectrum.

Figure 1 shows the $\mathrm{O} 1 s$ core-level spectra recorded (a) $15 \mathrm{~min}$ and (b) $205 \mathrm{~min}$ after exposing the $\mathrm{Si}(111)-(7 \times 7)$ surface to 20 Langmuir (L) of $\mathrm{O}_{2}$. One Langmuir corresponds to a $1 \mathrm{sec}$ exposure at a pressure of $1 \times 10^{-6}$ Torr. The two spectra are normalized using the background intensities, which are proportional to the photon flux. A large peak with an asymmetric tail at the higher binding-energy side is clearly observed at $531.7 \mathrm{eV}$ in Fig. 1(a). Three structures are also observed at $527.3,530.4$, and $534.9 \mathrm{eV}$. The spectrum in Fig. 1(a) looks different from the spectra shown in the previous $\mathrm{O} 1 s$ core-level reports, ${ }^{3,9}$ in which the peak at 534.9 $\mathrm{eV}$ was not observed. The structure at $530.4 \mathrm{eV}$ was not clear and the tail of the main peak extended to a binding energy larger than $536 \mathrm{eV}$ in the previous studies. This difference might come from the lower resolution in their experimental setups. In Fig. 1(b), the intensities of the 527.3-, 530.4-, and $534.9-\mathrm{eV}$ peaks decrease, and a new structure appears at $533.2 \mathrm{eV}$ along with an increase in intensity of the asymmetric tail of the 531.7-eV peak. To obtain information about the asymmetric tail, we have analyzed the spectra by a standard least-squares-fitting method using Voigt line shapes. The solid lines overlapped with the dots are the fitting results. A Gaussian width of $875 \mathrm{meV}$ full width at half maximum (FWHM) and a $200 \mathrm{meV}$ FWHM for the Lorentzian contribution were used for all $\mathrm{O} 1 s$ components that are shown below each spectrum. From the fitting results, we realize that the asymmetric tail originates from two components at 532.3 and $533.2 \mathrm{eV}$. Among the six O $1 \mathrm{~s}$ components, the three at $527.3,530.4$, and $534.9 \mathrm{eV}$ have finite lifetimes, which indicate that they originate from the metastable oxygen. Different behaviors are observed for the three other components, i.e., the intensities of the 532.3- and 533.2-eV components increase, while there is hardly any change in intensity with the 531.7-eV component. Here, we notice that the relative intensity of the three metastable components shows a photon
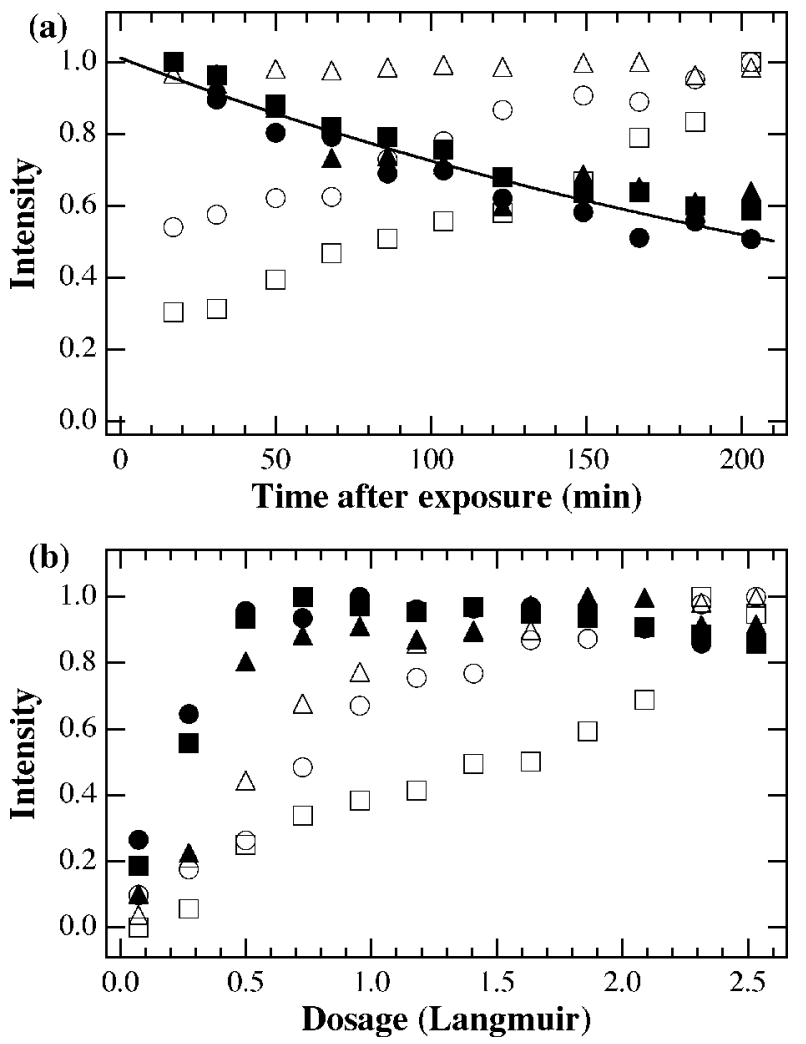

FIG. 2. (a) Time-dependent and (b) dosage-dependent intensities of each $\mathrm{O} 1 s$ component. The initial exposure is $20 \mathrm{~L}$ in (a). The intensity of each component is normalized with its maximum intensity in (a) and (b). The marks correspond to the components indicated in Fig. 1.

energy dependence, e.g., the intensity ratio of the 527.3-, 530.4-, and 534.9-eV components was approximately 2:3:1 at a photon energy of $665 \mathrm{eV}$, and 1:2:1 at $644 \mathrm{eV}$. Thus, due to the diffraction effect, the relative intensity of the $\mathrm{O} 1 \mathrm{~s}$ components in each spectrum of Fig. 1 does not represent the relative number of the oxygen atoms.

In order to determine the origins of the $\mathrm{O} 1 \mathrm{~s}$ components, we have measured the time-dependent and the dosagedependent core-level spectra. Figure 2(a) displays the timedependent intensity of each $\mathrm{O} 1 \mathrm{~s}$ component after a $20 \mathrm{~L}$ exposure, and (b) shows the dosage dependence. The marks used in Fig. 2 correspond to the components labeled by the same marks in Fig. 1. The intensity of each component, which is obtained by fitting all spectra, is normalized to its maximum intensity in each figure. From Fig. 2(a), we realize that the three metastable components show the same timedependent decrease. By fitting the decrease in intensity using the equation $y=\exp (-x / \tau)$ we can derive the lifetime $(\tau)$ of the metastable components. The solid line shown in Fig. 2(a) is obtained using $\tau=300$ as a parameter, which indicates that the lifetime is $300 \mathrm{~min}$ for all three metastable components. Regarding the three other components, the time-dependent increases in the intensities of the 532.3- and 533.2-eV components, and the constant intensity of the $531.7-\mathrm{eV}$ component are clearly observed in Fig. 2(a).

To obtain the dosage dependence shown in Fig. 2(b), we continuously measured photoemission spectra of the $\mathrm{Si}(111)$ 
(a)
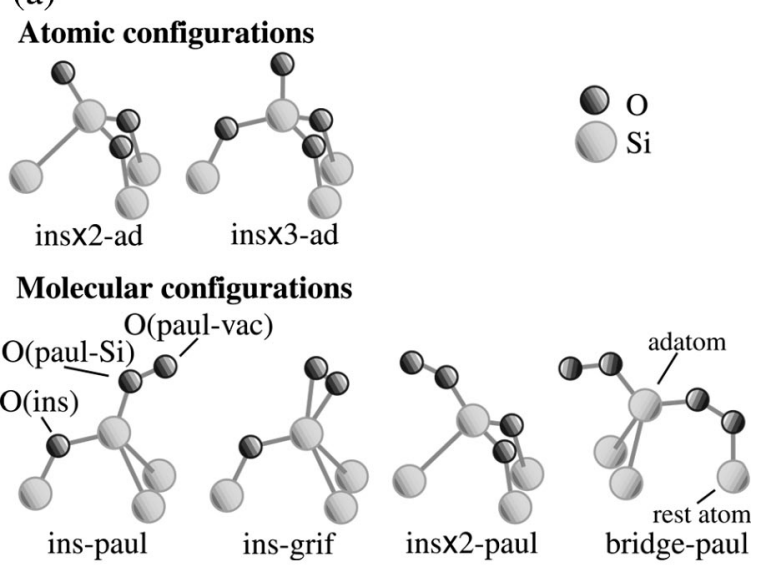

(b)

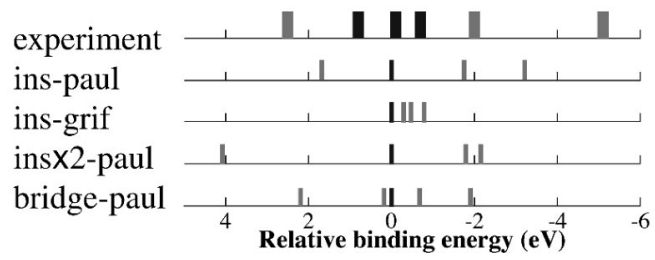

FIG. 3. (a) Schematic illustrations of the bonding configurations for the metastable oxygen on a $\mathrm{Si}(111)-(7 \times 7)$ surface. (b) Experimental and calculated binding energies of the $\mathrm{O} 1 \mathrm{~s}$ core level. The black and gray lines indicate the binding energies of the $\mathrm{O} 1 \mathrm{~s}$ components that originate from the stable and metastable bonding configuration, respectively.

surface exposed to an ambient $\mathrm{O}_{2}$ pressure of $7.5 \times 10^{-10}$ Torr. As displayed in the figure, the intensities of the 527.3-, 530.4-, and 534.9-eV components drastically increase in the beginning of the exposure and saturate at approximately 0.5 $\mathrm{L}$, whereas the intensities of the other $\mathrm{O} 1 s$ components continuously increase within the dosage range shown in Fig. 2(b). Taking into account that the 527.3-, 530.4-, and 534.9-eV components have the same lifetime and that their intensities saturate at the same dosage, these three components are concluded to originate from one and the same bonding configuration. Furthermore, the difference in binding energy reveals that at least three oxygen atoms with rather different chemical environments contribute to the bonding configuration of the metastable oxygen.

Figure 3(a) shows the possible bonding configurations of the metastable oxygen. Here, we only consider the two atomic configurations that contain more than two oxygen atoms among the configurations expected from the DFT calculations. ${ }^{1,2}$ The four molecular configurations that are proposed in the recent studies ${ }^{18-21}$ will also be discussed. The "ad" denotes an $\mathrm{O}$ atom bonding on top of a Si adatom of the dimer-adatom-stacking fault (DAS) structure ${ }^{22}$ of the $\mathrm{Si}(111)-(7 \times 7)$ surface, and "ins" corresponds to an $\mathrm{O}$ atom inserted into its back bond. The difference between the "paul" and "grif" configurations is the different number of $\mathrm{O}-\mathrm{Si}$ bonds between the adatom and the molecular oxygen, i.e., "paul" has only one O-Si bond and "grif" has two bonds. The "bridge" means an oxygen molecule adsorbed between an adatom and a rest atom of the DAS structure. Taking the symmetry of the bonding configurations into ac- count, we realize that the oxygen atoms adsorbed into the back bond have equivalent chemical environments within each configuration. Oxygen atoms with equivalent chemical environments should have core levels with the same binding energy, and thus the atomic configurations can reproduce only two $\mathrm{O} 1 \mathrm{~s}$ components. An observation of only two $\mathrm{O} 1 s$ components, with rather different binding energies, is also predicted by the DFT calculation ${ }^{2}$ for the atomic configurations. This is in obvious contrast with our experimental findings. The presence of three metastable components instead indicates that the major configuration of the metastable oxygen is a molecular one at $100 \mathrm{~K}$.

To discriminate between the four molecular configurations, we have calculated the binding energies of the $\mathrm{O} 1 \mathrm{~s}$ core levels of each oxygen atom. A $(2 \times 2)$ unit cell that contains one adatom and one rest atom, and a discrete variational $\mathrm{X} \alpha$ method in the linear combination of atomic orbitals (LCAO) representation ${ }^{23}$ are used in the calculation procedure. The calculated binding energies of the oxygen atoms of the three molecular configurations are shown in Fig. 3(b) together with the experimental result. The black and gray lines indicate the binding energies of the $\mathrm{O} 1 s$ components that originate from the stable and metastable bonding configurations, respectively. For the experimental results the zero of the relative binding-energy scale was chosen to coincide with the average energy of the three components, which are not related to the metastable oxygen. The theoretical results were aligned such that the binding energy of the $\mathrm{O} 1 s$ core level in the ins $\times 3$ configuration is zero. As shown in Fig. 3(b), the calculated binding energies of the ins-paul configuration show better agreements with those obtained experimentally, than those of the three other configurations. Hence, we conclude that the structure of the metastable species adsorbed on a $\mathrm{Si}(111)-(7 \times 7)$ surface is the ins-paul configuration. Further, comparing the experimental and calculated binding energies, the 527.3-, 530.4-, and $534.9-\mathrm{eV}$ components are concluded to originate from the $\mathrm{O}$ (paul-vac), O (ins), and $\mathrm{O}$ (paul-Si) atoms, respectively.

Finally, we discuss briefly the origins of the three $\mathrm{O} 1 \mathrm{~s}$ components observed at 531.7, 532.3, and $533.2 \mathrm{eV}$, and the oxidation process that occurs through the metastable oxygen. Oxygen atoms are reported to first adsorb into the back bonds of the adatoms and then on top of them by scanning tunneling microscopy (STM). ${ }^{12-15}$ The STM results indicate that the atomic oxygen species has the "ins $\times n$-ad" or "ins $\times n "(n=1,2$, and 3$)$ configuration on a $\operatorname{Si}(111)-(7 \times 7)$ surface. Each ins $\times n$-ad configuration is expected to have two $\mathrm{O} 1 s$ components with rather different binding energies $(>2.5 \mathrm{eV})$ by the DFT calculation. ${ }^{2}$ Every ins $\times n$ configuration is suggested to have one $\mathrm{O} 1 \mathrm{~s}$ component, whose binding energy varies for different $n$ within a range of 1.2 $\mathrm{eV}^{2}$ Taking into account that the three components are observed within a binding-energy range of $1.5 \mathrm{eV}$, the 531.7-, $532.3-$, and 533.2-eV components are supposed to originate from the ins $\times n$ configurations. The decreases in intensities of the metastable components are accompanied by increases in the intensities of the 532.3- and 533.2-eV components, and thus most of the molecular oxygen does not desorb from the surface. Instead the bonding configuration changes into 
stable ones after a certain time, producing the two $\mathrm{O} 1 \mathrm{~s}$ components. The constant intensity of the 531.7-eV component shows that the decay of the metastable species does not produce this component. From the ins-paul configuration, the ins configuration can be only produced by the desorption of the oxygen molecule. On the other hand, the metastable species can change its bonding configuration into the ins $\times 3$ configuration without any oxygen desorption, and into ins $\times 2$ with the desorption of one oxygen atom. Thus, although it is hard to determine their origins based on the present result alone, we propose that the 531.7-eV component originates from the ins configuration, and the 532.3- and 533.2-eV components from the ins $\times 2$ and ins $\times 3$ configurations. This proposition implies that the main oxidation process of the $\mathrm{Si}(111)$ surface is clean $\rightarrow$ ins $\rightarrow$ ins-paul $\rightarrow$ ins $\times 2$ and ins $\times 3$.

In conclusion, we have studied the metastable oxygen adsorbed on a $\mathrm{Si}(111)-(7 \times 7)$ surface using real time high- resolution $\mathrm{O} 1 \mathrm{~s}$ core-level measurements at $100 \mathrm{~K}$. After exposing the $\mathrm{Si}(111)$ surface to $\mathrm{O}_{2}$, six $\mathrm{O} 1 s$ components are observed at 527.3, 530.4, 531.7, 532.3, 533.2, and 534.9 eV. The 527.3-, 530.4-, and 534.9-eV components that originate from the metastable oxygen, have the same lifetime (300 min) and their intensities saturate at the same dosage. These results and the calculated binding energies of the O $1 s$ lead to a conclusion that the metastable molecular species has the ins-paul configuration. Moreover, the 527.3-, 530.4-, and 534.9-eV components are concluded to originate from the $\mathrm{O}$ (paul-vac), $\mathrm{O}$ (ins), and $\mathrm{O}$ (paul-Si) atoms, respectively.

Experimental support from Dr. T. Balasubramanian and the MAX-lab staff are gratefully acknowledged. This work was financially supported by the Swedish Research Council and the Carl Trygger Foundation (K.S.). H.W.Y. was supported by KOSEF through ASSRC, BK21 program and Teralevel Nano Device project of 21st Century Frontier program.

*Electronic address: kazsa@ifm.liu.se

1 S.-H. Lee and M.-H. Kang, Phys. Rev. Lett. 82, 968 (1999); Phys. Rev. B 61, 8250 (2000).

${ }^{2}$ S.-H. Lee and M.-H. Kang, Phys. Rev. Lett. 84, 1724 (2000).

${ }^{3}$ U. Höfer, P. Morgen, W. Wurth, and E. Umbach, Phys. Rev. Lett. 55, 2979 (1985); Phys. Rev. B 40, 1130 (1989).

${ }^{4}$ P. Morgen, U. Höfer, W. Wurth, and E. Umbach, Phys. Rev. B 39, 3720 (1989).

${ }^{5}$ P. Bratu, K.L. Kompa, and U. Höfer, Phys. Rev. B 49, 14070 (1993).

${ }^{6}$ G. Dujardin, G. Comtet, L. Hellner, T. Hirayama, M. Rose, L. Philippe, and M.J. Besnard-Ramage, Phys. Rev. Lett. 73, 1727 (1994).

${ }^{7}$ G. Comtet et al., Surf. Sci. 331-333, 370 (1995); ibid. 352-354, 315 (1996).

${ }^{8}$ K. Sakamoto et al., Surf. Sci. 306, 93 (1994); ibid. 357/358, 514 (1996).

${ }^{9}$ B. Lamontagne, D. Roy, R. Sporken, and R. Caudano, Prog. Surf. Sci. 50, 315 (1995).

${ }^{10}$ H. Ibach, H.D. Bruchmann, and H. Wagner, Appl. Phys. A: Solids Surf. 29, 113 (1982).

${ }^{11}$ J.P. Pelz and R.H. Koch, Phys. Rev. B 42, 3761 (1990); J. Vac. Sci. Technol. B 9, 775 (1991).

${ }^{12} \mathrm{Ph}$. Avouris, I.-W. Lyo, and F. Bozso, J. Vac. Sci. Technol. B 9, 424 (1991).

${ }^{13}$ I.-W. Lyo, Ph. Avouris, B. Schubert, and R. Hofmann, J. Phys. Chem. 94, 4400 (1990).

${ }^{14}$ R. Martel, Ph. Avouris, and I.-W. Lyo, Science 272, 385 (1996).

${ }^{15}$ G. Dujardin, A. Mayne, G. Comtet, L. Hellner, M. Jamet, E. Le Goff, and P. Millet, Phys. Rev. Lett. 76, 3782 (1996).

${ }^{16}$ I.-S. Hwang, R.-L. Lo, and T.T. Tsong, Phys. Rev. Lett. 78, 4797 (1997); Surf. Sci. 399, 173 (1998).

${ }^{17}$ K.-Y. Kim, T.-H. Shin, S.-J. Han, and H. Kang, Phys. Rev. Lett. 82, 1329 (1999).

${ }^{18}$ T. Hoshino and Y. Nishioka, Phys. Rev. B 61, 4705 (2000).

${ }^{19}$ K. Sakamoto, S. Doi, Y. Ushimi, K. Ohno, H.W. Yeom, T. Ohta, S. Suto, and W. Uchida, Phys. Rev. B 60, R8465 (1999).

${ }^{20}$ F. Matsui, H.W. Yeom, K. Amemiya, K. Tono, and T. Ohta, Phys. Rev. Lett. 85, 630 (2000).

${ }^{21}$ T. Jensen, L.-B. Taekker, C. Gundlach, F.K. -Dam, P. Morgen, S.V. Hoffman, Z. Li, and K. Pedersen, Phys. Rev. B 64, 045304 (2001).

${ }^{22}$ K. Takayanagi, Y. Tanishiro, M. Takahashi, and S. Takahashi, Surf. Sci. 164, 367 (1985).

${ }^{23}$ D.E. Ellis and G.S. Painter, Phys. Rev. B 2, 887 (1970). 